\title{
Curcumin: A Magnificent Therapeutic Molecule from Traditional Medicinal System Raghav N*
}

Department of Chemistry, Kurukshetra University, Haryana, India

\section{Editorial}

Natural products, in general are rich source of potentially active therapeutic drugs but optimization of active content and their recognition as therapeutic design with mode of action on molecular targets to become useful pharmacological agents need a special focus for their acceptance in elite society and medical approval. With increasing reports of side effects in synthetic drugs, naturally occurring substances found in plants have generated considerable public and scientific interests for the use of phytochemicals derived from dietary components to combat human diseases. Curcumin, a yellowish orange compound chemically known as 1,6-heptadiene-3,5-dione-1,7-bis(4hydroxy-3-methoxy phenyl)-(1E,6E) also called diferulolylmethane, an active ingredient of the spice turmeric processed from rhizome of Curcuma longa, used in India and other regions of Asia, has a long history as an herbal remedy used in Indian and Chinese traditional medicinal system as early as $700 \mathrm{AD}$ for a variety of diseases. Curcumin was predominantly used to treat colds, parasitic worms, leprosy, inflammatory conditions including bronchitis, arthritis, inflammations of bladder, liver and kidney, urinary tract infections, skin disease, and in symptoms such as fever and diarrhea.

Multiple therapeutic activities such as anti-inflammatory, antioxidant and anticancer are known to be possessed by this molecule. Curcumin has been ascribed a multitude of therapeutic activities and has also been associated with angiogenesis, tumourigenesis, hepatic fibrosis, diabetes and with therapeutic effects in diseases of the cardiovascular, pulmonary, neurological, gastrointestinal systems and of skin and liver. The pharmacological effect is exerted through variety of mechanistic aspects including interaction with receptors, enzymes or other molecular targets that contribute toward diseased conditions. Curcumin has diverse probability of interactions with the biomolecules because of its structure. It can exist in enolic and diketonic forms. Being symmetrical molecule, the conjugated system of any direction can be accessible to biomolecules without any steric restriction and may be one of the reasons of its interaction with variety of molecular targets (Figure 1).

Curcumin is an effective scavenger of reactive oxygen or nitrogen species. Scavenging properties of curcumin have also been considered to be responsible for its protective role against oxidative damage of DNA and proteins believed to be associated with a variety of chronic diseases such as cancer, atherosclerosis, neurodegenerative diseases and ageing. The structure possesses stable conjugated system and phenolic hydroxyl groups which are important for curcumin's antioxidant activities. This scaffold contributes to anti-oxidant property of curcumin and acts as sink of free radicals and hence scavenges their untoward effects.

The linker and its carbonyl functions are important for antiinflammatory activity. The anti-inflammatory activity of curcumin has been found comparable to steroidal drugs and non-steroidal anti-inflammatory drugs such as indomethacin and phenylbutazone. Curcumin is known to decrease the inflammation associated with experimental colitis, including a substantial reduction of the rise in myleoperoxidase activity, an established marker for inflammatory cells mainly polymorphonuclear leukocytes. It also suppresses prostaglandin synthesis by its inhibitory effect on cyclooxygenase, a key enzyme responsible for the conversion of arachidonic acid

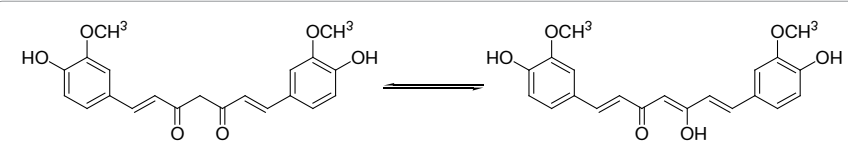

Figure 1: Curcumin molecule.

to prostaglandins. The lysosomal cysteine proteinases, Cathepsins which play an important role in intracellular protein degradation and recently reported to play a role in apoptosis along with caspases are also inhibited by curcumin to an appreciable extent, contributing toward anti-arthritic, anti-Alzheimer and anti-cancer properties of curcumin. Curcumin exerts its inhibitory effect on cancer development by several mechanisms such as inhibition of carcinogen activation and stimulation of carcinogen detoxification, prevention of oxidative DNA damage and its capacity to reduce inflammation as well. Two $\sigma, \beta$ unsaturated moieties leading to bis-conjugated enone structure, present in the molecule have been shown to act as Michael acceptors may be responsible for curcumin's anti-cancer activity rendering it accessible to various nucleophilic groups of enzymes leading to enzyme inhibition. Consider any mechanistic aspect of anticancer agent; curcumin has been found capable to execute most of these effectively. Curcumin has also been shown to suppress the production of cytokines such as interferon, interleukins and tumour necrosis factor; to inhibit the inducible nitric oxide synthase; and to suppress the activation of the nuclear transcription factor. Curcumin inhibits cell proliferation of a variety of transformed cell types, including T- and B cell lymphomas, breast, colon, gastric, ovarian, prostate and oral epithelial carcinoma cells. Proteasome activity is also inhibited in presence of curcumin which, exhibits changes in cell cycle progression and induces apoptosis in different human colon, ovarian carcinoma cell line. Cells growth inhibition, cell cycle arrest in G2/M phase has also been observed. It offers effective therapy against cisplatin resistant ovarian cancer cells. Curcumin is capturing the attention of cancer investigators worldwide because of its potent inhibitory properties against human malignancies. It induces apoptotic cell death in human promyelocytic leukaemia cells and human oral squamous carcinoma cells. Curcumin also shows a potent anticarcinogenic activity against a broad range of tumours, including skin, fore stomach, duodenal and colon carcinogenesis. Curcumin was found to be a potent cytotoxic agent against bladder tumour cell lines and human prostate cancer cells. A wide variety of pharmacological activity viz., antibacterial, antifungal, antiviral, antiHIV, anti-Parkinson's, anti-angiogenesis, antirheumatic, antimalarial,

*Corresponding author: Raghav N, Professor, Department of Chemistry, Kurukshetra University, Haryana, India, Tel: 09896918277; E-mail: nraghav.chem@gmail.com

Received: December 01, 2015; Accepted: December 03, 2015; Published December 07, 2015

Citation: Raghav N(2015) Curcumin:AMagnificent Therapeutic Molecule from Traditional Medicinal System. Biochem Anal Biochem 4: e160. doi:10.4172/2161-1009.1000e160

Copyright: (C) 2015 Raghav N. This is an open-access article distributed under the terms of the Creative Commons Attribution License, which permits unrestricted use, distribution, and reproduction in any medium, provided the original author and source are credited. 
antiprotozoan, wound treatment, hepatoprotective activity, antileishmanial activity and amyloid heart disease have also been reported for curcuminoid and its derivatives [1,2].

Hepatoprotective activity can also be due to alkaline phosphatase inhibition potency of curcumin, an enzyme involved in various conditions such as cholecystitis, cholestasis, cholangitis, cirrhosis, hepatitis, fatty liver, liver tumour, liver metastasis, drug intoxication, Paget's disease, osteosarcoma, bone metastasis, prostatic cancer, renal osteodystrophy, fractured bone, osteomalacia and others such as policythemiavera, myelofibrosis, seminoma etc. It seems that the magnificent molecule knows where to react. A usual thinking is that any molecule with nucleophilic center will necessarily act as inhibitor of every enzyme, but curcumin does not affect acid phosphatase activity which has been reported in diminished state in prostate cancer [1,2].

In the case of curcumin, the poor aqueous solubility and relatively low bioavailability have been major obstacles for its clinical development as a therapeutic drug, but the same has contributed towards its efficacy in the diseases of gastrointestinal tract. This chemical has proved to be an excellent synthone and has diverse possibility of modification that may result in more effective therapeutic molecule.

Because curcumin interacts with such a wide range of target proteins there is a need to determine to which specific structural motif curcumin binds. Identification of such specific binding motifs of proteins are important to understand biological role of curcumin and is a major step in developing more specific and effective curcumin analogs of therapeutic interest. The studies can result in better understanding of molecular target-curcumin binding phenomenon. To conclude, the molecule from traditional medicinal system has emerged as potential therapeutic molecule with modern pharmacological explanation and provides options of further research in various fields.

\section{References}

1. Ravish I, Raghav N (2014) Curcumin as inhibitor of mammalian Cathepsin B Cathepsin $\mathrm{H}$, acid phosphatase and alkaline phosphatase: a correlation with pharmacological activities. Med Chem Res 23: 2847-2855.

2. Rajasekaran SA (2011) Therapeutic potential of curcumin in gastrointestinal diseases. World. J Gastrointest Pathophysiol 2: 1-14. 